\title{
INSECTOS FITÓFAGOS EN PEJIBAYE (Bactris gasipaes K.) PARA PALMITO ${ }^{1}$
}

\author{
Carlos Arroyo Oquendo ${ }^{2}$, Ramón G. Mexzón ${ }^{3}$, Jorge Mora Urpi ${ }^{4}$
}

\begin{abstract}
RESUMEN
Insectos fitófagos en pejibaye (Bactris gasipaes K.) para palmito. Las poblaciones de los insectos fitófagos asociados al pejibaye (Bactris gasipaes) destinado para la producción de palmito, fueron evaluadas en una prueba de variedades en una localidad de la vertiente caribeña de Costa Rica. Nueve especies de insectos fueron identificados: a. taladradores del tallo: Metamasius hemipterus y Rhynchophorus palmarum; b. defoliadores: Conocephalus sp., Neoconocephalus sp., Schistocerca pos. nitens y Saliana pos. severus, y c. raspadores de follaje: Demotispa pos. pallida y Cephaloleia sp. En forma adicional, se hizo un conteo de nidos de la hormiga Solenopsis geminata, la cual se asocia con la base del tallo. Ninguna de las especies alcanzó la condición de plaga y sus poblaciones se mantienen estables. El estudio que abarcó los primeros 12 meses de cultivo, mostró que los insectos tuvieron una mayor abundancia relativa en las variedades sin espinas en comparación con la variedad con espinas, de uso tradicional en plantaciones comerciales. Bajo condiciones de estrés para la planta es posible que alguna de esas especies se convierta en plaga, por lo tanto se recomienda el monitoreo de plagas.
\end{abstract}

Palabras claves: insectos, plagas, pejibaye, Bactris gasipaes, palmito.

\begin{abstract}
Phytophagous insects in peach palm (Bactris gasipaes K.) grown for heart of palm production. An evaluation of the populations of phytophagous insects present in a variety trial of peach palm for heart of palm productions, was carried out in a locality of the Caribbean region of Costa Rica. Nine species of insects were identified: a. stem borers: Metamasius hemipterus y Rhynchophorus palmarum; b. defoliators: Conocephalus sp., Neoconocephalus sp., Schistocerca pos. nitens y Saliana pos. severus, and c. foliar rasping insects: Demotispa pos. pallida y Cephaloleia sp. In addition, the nests of the ant Solenopsis geminata associated with the basal section of the stem were counted. None of those species reached a level of economic importance and their populations did not show important changes throughout the time of the study. But, during those 12 months the relative abundance of the insects was higher in the spineless varieties than in the spiny one, which is the traditional commercial variety. Some of those insects could become a plague under unusual environmental changes which stress the plants and favor their multiplication. For that reason it is convenient to monitor them to avoid surprises.
\end{abstract}

Key words: insects, plagues, peach palm, Bactris gasipaes, heart of palm.

\section{INTRODUCCIÓN}

El conocimiento de aspectos básicos de la dinámica poblacional de insectos fitófagos así como su comportamiento fenológico durante el desarrollo del pejibaye, es fundamental para un manejo eficiente de las especies nocivas; esto es particularmente palpable en una siembra de pejibaye para producción de palmito, donde muchas especies de insectos se asocian a los cortes frescos en los tallos, derivados de la extracción del palmito.

En este cultivo existen pocos estudios de los organismos insectiles que pueden causar daño. El primer trabajo fue realizado en Colombia, el cual informó la presencia de 15 especies de insectos, ninguno de ellos causando daño económico en el cultivo (Pava et al.

\footnotetext{
1 Recibido para publicación el 15 de marzo del 2004. Proyecto financiado parcialmente por FITTACORI.

2 Escuela de Zootecnia. Universidad de Costa Rica. Correo electrónico: carroyo@cariari.ucr.ac.cr

3 Escuela de Agronomía. Universidad de Costa Rica.

4 Escuela de Biología. Universidad de Costa Rica
} 
1981, citado por Velazco 1991). También, Mora et al. (1982) hacen referencia de Metamasius hemipterus L. (Curculinidae), Rhynchophorus palmarum L. (Curculinidae) y Strategus aloeus L. (Scarabaidae) como plagas potenciales y mencionan otros artrópodos que afectan el pejibaye en Costa Rica. Rothschuh (1983) informó que los escarabajos $M$. hemipterus y $R$. palmarum. fueron los insectos que más daños ocasionaban en el tallo del pejibaye en la Estación Experimental El Recreo en Nicaragua. Asimismo, Couturier et al. (1991) informaron que el pejibaye sembrado como monocultivo sufrió del síndrome de "caída de los frutos" y se debió, entre otros factores, al chinche Coreidae, Leptoglossus lonchoides Allen, en la Amazonia Central (Brasil).

Mexzón (1999), informó de 35 especies perjudiciales de plagas insectiles en la zona caribeña de Costa Rica, y señaló que los síntomas y los daños de importancia económica fueron causados por los escarabajos Demotispa pos. pallida Dally (Chrysomelidae) y M. hemipterus. Otros insectos que afectaron en menor grado fueron las especies Cephaloleia sp. (Chrysomelidae) raspando el follaje y $R$. palmarum afectando el tallo.

El mismo autor (1999) manifestó que algunos insectos podrían convertirse en una plaga cuando se siembren plantaciones comerciales de pejibaye sin espinas, por lo tanto, para su regulación, habrá que considerar la integración de varias medidas de combate.

Más recientemente, Vásquez et al. (2000) informaron que el escarabajo Dynamis nitidulus (Curculinidae), provocó daños similares a los ocasionados por $R$. palmarum en plantaciones de pejibaye para palmito en la región de Iquitos, Amazonía Peruana. También, O’Brien y Kovarik (2000) informan del escarabajo curculiónido, Palmelampius heinrichi O’Brien, cuyas hembras causan serios daños en los frutos de pejibaye en Brasil, Colombia, Ecuador y Perú.

El objetivo del presente trabajo fue, comparar la abundancia relativa de insectos fitófagos en cuatro variedades de pejibaye para palmito: tres sin espinas ( $U t i$ lis-Guatuso, Diamantes 10 y Tuira-Darién) y una con espinas (Utilis-Tucurrique), durante los primeros 12 meses de crecimiento del cultivo en Guápiles, Limón, Costa Rica.

\section{MATERIALES Y MÉTODOS}

\section{Área de estudio:}

El ensayo se realizó de mayo del 2000 a abril del 2001 en el área experimental de pejibaye que mantiene la Universidad de Costa Rica bajo el convenio INTA-
UCR, en la Estación Experimental Los Diamantes, ubicada en Guápiles, cantón Pococí, provincia de Limón. La posición geográfica es $10^{\circ} 13^{\prime}$ latitud norte y $86^{\circ}$ 46' longitud oeste, y la altitud es de $249 \mathrm{msnm}$. Durante el ensayo la temperatura promedio fue de $25,9^{\circ} \mathrm{C}$, con una mínima de $23,9^{\circ} \mathrm{C}$ en abril y una máxima de $28^{\circ} \mathrm{C}$ en noviembre; la precipitación acumulada fue de $4.065 \mathrm{~mm}$, con un promedio mensual de 338,9 mm (Figura 1). El viento soplaba del NE.
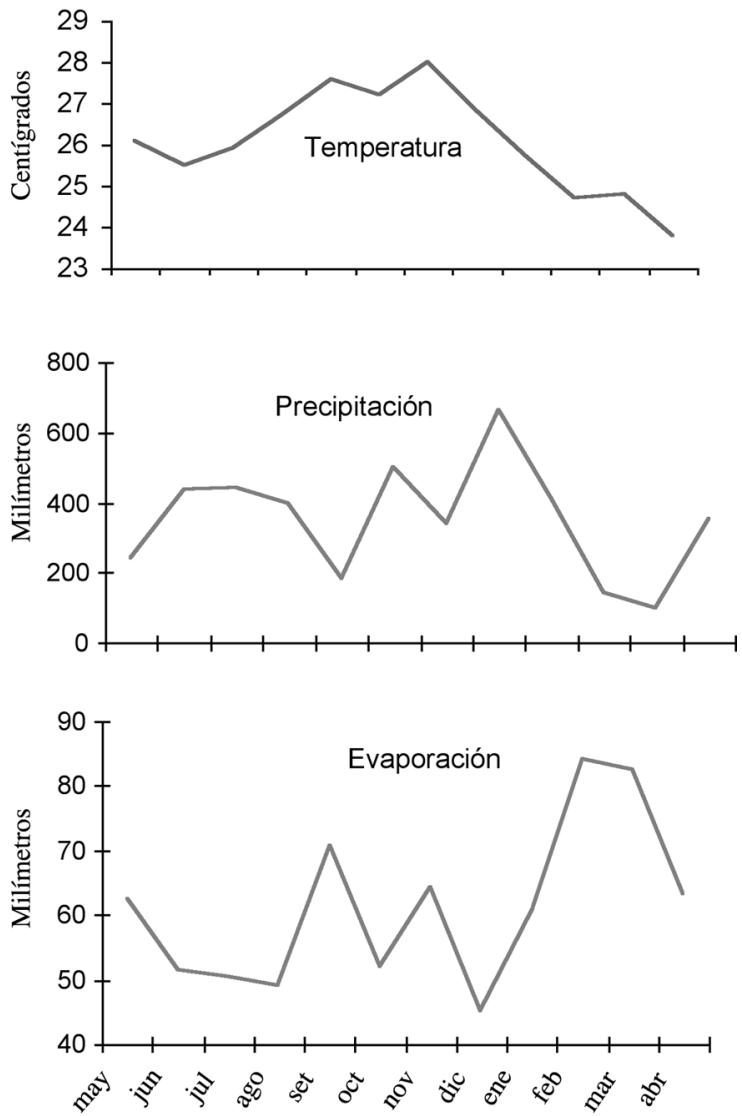

Figura 1. Datos climatológicos de temperatura, precipitación y evaporación en el período experimental (promedios mensuales) en Guápiles, 2000-2001.

Previo a la preparación del terreno el área estaba cubierta por Paspalum fasciculatum Will. (Poaceae) en un $95 \%$ y un $5 \%$ por especies de hoja ancha, principalmente Sida spp., Mimosa pudica L. (Leguminosae) y Colocasia esculenta (Araceae). Esta vegetación tenía una altura promedio de $70 \mathrm{~cm}$. Un mes después de la siembra brotaron en poca cantidad, Ageratum conyzoides L. (Asteraceae), C. esculenta, M. pudica, Commelina diffusa L. (Commelinaceae)., Cyperus sp. (Cyperaceae), esta situación se mantuvo constante durante el primer año de desarrollo del pejibaye. La vegetación aledaña al ensayo fue diversa, incluyendo poáceas, 
asteráceas, ciperáceas, euforbiáceas, leguminosas, rubiáceas, malváceas, solanáceas y otras. Al lado norte, se encontraba un bloque de pasto King grass (Pennisetum purpureum L.) (Poaceae), al sur había pejibaye para palmito, donde se incluía un ensayo de control de malezas (Bogantes 2003), al oeste estaba un potrero bajo pastoreo y al oeste, un bosque primario, compuesto de eufobiáceas y leguminosas. La vegetación herbácea y arbustiva era de Ipomoea spp. (Convolvulaceae), Vitis spp. (Vitaceae) y Hamelia patens Jacquin (Rubiaceae).

\section{Manejo experimental}

La plantación se estableció con plántulas recién germinadas (dos hojas abiertas), de cuatro variedades: tres sin espinas, Diamantes-1 (de ascendencia UtilisGuatuso), Diamantes-10 (de ascendencia Yurimaguas) y Diamantes-20 (de ascendencia Tuira - Darién); y una con espinas (Utilis-Tucurrique). El diseño experimental fue un irrestricto al azar con cuatro tratamientos (variedades) y cinco repeticiones por tratamiento para una densidad de siembra de 10.000 plantas por hectárea. El tamaño de la parcela fue de 45 plantas útiles, dispuestas a 2,0 m entre hileras y $0,5 \mathrm{~m}$ entre plantas, para un total de 225 plantas por tratamiento (variedad).

Previo a ello, el terreno se aró y rastreó. Se aplicó un herbicida premergente selectivo, alaclor $\left(\operatorname{Lazo}^{\circledR}\right)$. Ocho días después de la siembra se encaló con carbonato de calcio y se aplicaron $30 \mathrm{~g}$ de fosfato diamónico por planta y en adelante, hasta los seis meses, se continuó fertilizando en forma mensual alternando con $30 \mathrm{~g} / \mathrm{plan}$ ta de nitrato de amonio y $30 \mathrm{~g}$ de la fórmula $18 \mathrm{~N}-5 \mathrm{P}$ $-15 \mathrm{~K}-6 \mathrm{Mg}-2 \mathrm{~B}-2 \mathrm{~S}$. Después de los seis meses se abonó en meses alternos con los mismos fertilizantes pero utilizando $60 \mathrm{~g} /$ planta. El combate de malas hierbas se realizó en forma integral utilizando control químico y físico, según se consideró necesario, basándose en las recomendaciones de Bogantes (2003).

Para efecto de este estudio, los insectos se contaron en forma visual en diez plantas por repetición, 50 plantas por variedad (tratamientos), luego se fijó el número promedio de insectos en 10 plantas. En forma complementaria se pasó la red entomológica a razón de 30 golpes de red por repetición.

El reconocimiento y cuantificación de los insectos capturados en el campo se realizó cada tres meses (julio, octubre, enero y abril) durante el primer año del cultivo. Cuando se tuvo duda en el reconocimiento de alguna especie se llevaron especimenes al laboratorio de insectos para ser identificados bajo un microscopio estereoscopio a $45 \mathrm{X}$. También se anotó el número de hormigueros presentes por repetición.

Para el análisis estadístico de los datos se usó el paquete estadístico SAS (2001). Las comparaciones de medias se realizaron con la prueba denominada Waller-Duncan, con una significancia $\mathrm{P}(\alpha \leq 0,05)$.

\section{RESULTADOS Y DISCUSIÓN}

Un total de 123 insectos fueron observados, los cuales se agruparon en ocho géneros de insectos fitófagos perjudiciales (Cuadro 1), los cuales se clasificaron, según su hábito de fitofagia: dos taladradores de tallo,

Cuadro 1. Número promedio de insectos capturados/10 plantas durante los primeros 12 meses de cultivo en cuatro variedades de pejibaye (Guápiles, Costa Rica. 2001).

\begin{tabular}{|c|c|c|c|c|c|c|c|c|c|}
\hline \multirow{3}{*}{$\begin{array}{l}\text { Especie de insecto } \\
\text { y tipo de fitofagia }\end{array}$} & \multicolumn{8}{|c|}{ Edad del palmito } & \multirow{3}{*}{ Total } \\
\hline & \multicolumn{2}{|c|}{3 meses } & \multicolumn{2}{|c|}{6 meses } & \multicolumn{2}{|c|}{9 meses } & \multicolumn{2}{|c|}{12 meses } & \\
\hline & c.e $e^{*}$ & s.e* & c.e & s.e & c.e & s.e & c.e & s.e & \\
\hline Taladradores de tallo & & & & & & & & & \\
\hline Metamasius hemipterus & 0,0 & 0,0 & 0,4 & 2,8 & 1,2 & 7,8 & 3,2 & 16,2 & 31,6 \\
\hline $\begin{array}{l}\text { Rhynchophorus palmarum } \\
\text { Defoliadores }\end{array}$ & 0,0 & 0,0 & 0,0 & 1,7 & 0,6 & 3,4 & 1,0 & 3,69 & 10,3 \\
\hline Neoconocephalus sp. & 0,2 & 0,6 & 1,2 & 6,4 & 0,4 & 5,6 & 0,4 & 2,0 & 16,8 \\
\hline Conocephalus sp. & 0,0 & 0,8 & 106 & 5,8 & 0,6 & 5,2 & 0,6 & 2,4 & 16,4 \\
\hline Schistocerca pos. nitens & 0,2 & 0,2 & 0,6 & 4,2 & 0,4 & 6,2 & 0,4 & 2,4 & 15,6 \\
\hline $\begin{array}{l}\text { Saliana } \text { sp. } \\
\text { Raspadores }\end{array}$ & 0,0 & 0,0 & 0,4 & 2,0 & 0,8 & 4,4 & 0,6 & 3,6 & 11,8 \\
\hline Cephaloleia sp. & 0,0 & 0,0 & 0,0 & 0,6 & 0,4 & 1,4 & 0,4 & 2,4 & 5,2 \\
\hline Demotispa pos. pallida & 0,0 & 0,0 & 0,6 & 4,0 & 0,8 & 4,4 & 0,8 & 4,8 & 15,4 \\
\hline
\end{tabular}

* c.e =palmito con espinas (Utlis-Tucurrique), s.e = palmito sin espinas (promedio de Diamantes-1, Diamantes-10 y Diamantes-20). 
dos raspadores de follaje y cuatro defoliadores (Figura 2). Además de la hormiga Solenopsis geminata. Estas especies están incluidas en los inventarios de artrópodos que atacan al pejibaye, informados por Mora et al. (1982); Rothschuh (1983); Velazco (1991) y Peña (2000). Sin embargo, este número de especies está muy por debajo del total de artrópodos perjudiciales que informa Mexzón (1995, 1999), debido, con alto grado de certeza, a la juventud y vigor de la plantación.

\section{Taladradores de tallo}

Se recolectaron un total de 42 escarabajos Curculinidae. De ellos, $32(75,4 \%)$ eran $M$. hemipterus y 10 (24,6 \%) R. palmarum. M. hemipterus es el principal escarabajo asociado con las lesiones en los tallos de pejibaye, ya que sus larvas taladran las bases de las vainas de las hojas, llegando incluso a dañar seriamente el ta- llo. $R$. palmarum es el principal vector del nematodo Bursaphelenchus cocophilus (Cobb), causante de la enfermedad conocida como anillo rojo que afecta algunas palmeras en la región tropical, pero no informadas aún en pejibaye, estudiado en detalle por Mexzón et al. (1994) y Chinchilla (2003).

Para ambos escarabajos su mayor abundancia relativa se presentó a partir de los seis meses y aumentó dinámicamente hasta los 12 meses, cuando se capturó la mayor cantidad de insectos (Cuadro 1), esta situación se debió a que ambas especies se alimentan y reproducen en los restos de tallos que quedaron en el campo después de cada cosecha. Los bloques de las variedades sin espinas (Diamantes-10, Diamantes-20 y Diamantes-1) presentaron una mayor vigorosidad, precocidad en el desarrollo y mayor producción de brotes (Cuadros 2, 3 y 4 (Arroyo y Mora 2003). Se colectó un mayor número de insectos que en la variedad con espinas Utilis-Tucurrique, que
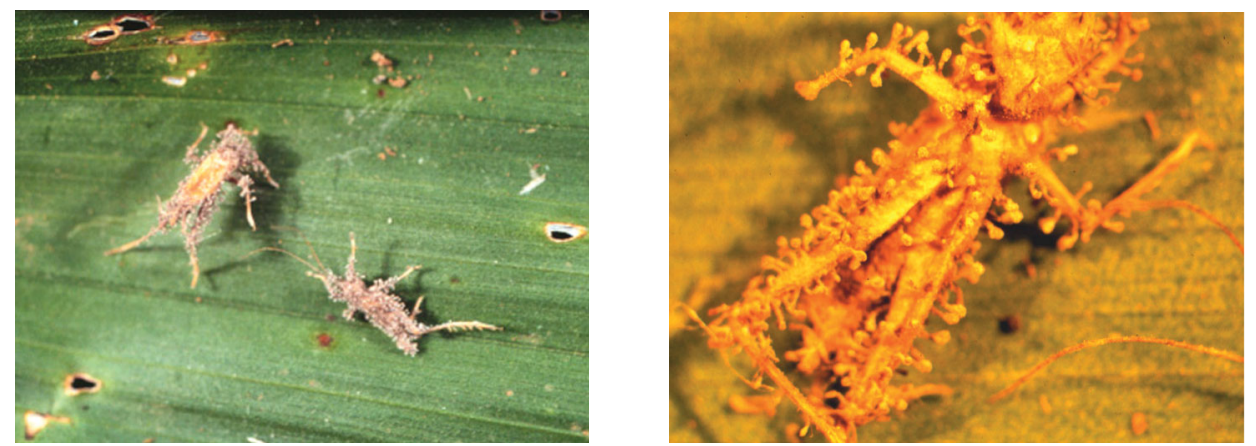

Figura 2. Saltamontes colonizado por Hirsutella sp.

Cuadro 2. Número promedio de insectos capturados/10 plantas, durante el primer año de crecimiento, en cuatro variedades de pejibaye (Guápiles, Costa Rica. 2001).

\begin{tabular}{lccccc}
\hline $\begin{array}{l}\text { Especie de insecto } \\
\text { Y tipo de fitofagia }\end{array}$ & Diamantes-1 & Diamantes-10 & Diamantes-20 & Utilis-Tucurrique & Total \\
\hline $\begin{array}{l}\text { Taladradores de tallo } \\
\text { Metamasius hemipterus }\end{array}$ & $6,8 \mathrm{~b}^{*}$ & $10,2 \mathrm{a}$ & $9,8 \mathrm{a}$ & $4,8 \mathrm{~b}$ & 31,6 \\
$\begin{array}{l}\text { Rhynchophorus palmarum } \\
\quad \text { Defoliadores }\end{array}$ & $2,3 \mathrm{a}$ & $3,4 \mathrm{a}$ & $3,0 \mathrm{a}$ & $1,6 \mathrm{~b}$ & 10,3 \\
$\begin{array}{l}\text { Neoconocephalus } \text { sp. } \\
\text { Conocephalus } \text { sp. }\end{array}$ & $4,2 \mathrm{a}$ & $4,8 \mathrm{a}$ & $5,6 \mathrm{a}$ & $2,2 \mathrm{~b}$ & 16,8 \\
$\begin{array}{l}\text { Schistocerca } \text { pos. } \text { nitens } \\
\text { Saliana } \text { sp. }\end{array}$ & $4,6 \mathrm{a}$ & $5,0 \mathrm{a}$ & $4,6 \mathrm{a}$ & $2,2 \mathrm{~b}$ & 16,4 \\
$\quad$ Raspadores & $4,2 \mathrm{a}$ & $4,4 \mathrm{a}$ & $5,4 \mathrm{a}$ & $1,6 \mathrm{~b}$ & 15,6 \\
Cephaloleia sp. & $3,8 \mathrm{a}$ & $4,0 \mathrm{a}$ & $2,2 \mathrm{~b}$ & $1,8 \mathrm{~b}$ & 11,8 \\
$\begin{array}{l}\text { Demotispa } \text { pos. } \text { pallida } \\
\text { Total }\end{array}$ & $1,0 \mathrm{~b}$ & $1,6 \mathrm{a}$ & $1,8 \mathrm{a}$ & $0,8 \mathrm{~b}$ & 5,2 \\
\hline
\end{tabular}

*a,b,c= Valores medios con letra distinta en líneas horizontales son estadísticamente diferentes, Duncan $(\mathrm{P} \leq 0,05)$. 
Cuadro 3. Valores promedio de algunas variables para el desarrollo de la planta de pejibaye en cuatro variedades de pejibaye, 12 meses después de la siembra (Tomado de Arroyo y Mora 2003).

\begin{tabular}{lrrrr}
\hline \multicolumn{1}{c}{ Variables } & \multicolumn{4}{c}{ Variedades } \\
\cline { 2 - 5 } & Diamantes 1 & Diamantes 10 & Diamantes 20 & Utilis-Tucurrique \\
\hline & $10,92 \mathrm{a}$ & $11,23 \mathrm{a}$ & $10,79 \mathrm{a}$ & $9,96 \mathrm{~b}$ \\
$\begin{array}{l}\text { Diámetro del tallo (cm) } \\
\text { Altura del tallo (cm) }\end{array}$ & $134,46 \mathrm{~b}$ & $152,30 \mathrm{a}$ & $133,34 \mathrm{~b}$ & $117,51 \mathrm{c}$ \\
$\mathrm{N}^{\circ}$ de hojas & $6,84 \mathrm{~b}$ & $7,28 \mathrm{ab}$ & $7,12 \mathrm{ab}$ & $7,52 \mathrm{a}$ \\
$\begin{array}{l}\text { Longitud vaina de la hoja } \\
\text { externa o de mayor edad (cm }\end{array}$ & $121,98 \mathrm{~b}$ & $146,94 \mathrm{a}$ & $119,63 \mathrm{~b}$ & $103,94 \mathrm{c}$ \\
$\begin{array}{l}\text { Longitud de la lámina de la } \\
\text { hoja externa(cm) }\end{array}$ & $167,80 \mathrm{a}$ & $178,22 \mathrm{a}$ & $171,40 \mathrm{a}$ & $142,04 \mathrm{~b}$ \\
$\begin{array}{l}\text { Longitud del pecíolo de la hoja } \\
\text { externa (cm) }\end{array}$ & $20,11 \mathrm{a}$ & $22,70 \mathrm{a}$ & $20,50 \mathrm{a}$ & $15,89 \mathrm{~b}$ \\
$\begin{array}{l}\text { Longitud total de la } \\
\text { hoja externa }(\mathrm{cm})\end{array}$ & $309,89 \mathrm{ab}$ & $347,86 \mathrm{a}$ & $311,53 \mathrm{ab}$ & $261,87 \mathrm{c}$ \\
\hline
\end{tabular}

* $\mathrm{a}, \mathrm{b}=$ Valores medios con letra distinta en líneas horizontales son estadísticamente diferentes, Duncan $(\mathrm{P} \leq 0,05)$.

Cuadro 4. Promedio de brotes o "hijuelos" por planta en cuatro variedades de pejibaye, durante los primeros 12 meses de desarrollo (observaciones hechas en plantas con raíz desnuda) (Tomado de Arroyo y Mora 2003).

\begin{tabular}{ccccc}
\hline \multirow{2}{*}{$\begin{array}{c}\text { Edad } \\
\text { (meses) }\end{array}$} & $\begin{array}{c}\text { Variedades } \\
\text { Tucurrique }\end{array}$ & $\begin{array}{c}\text { Diaman- } \\
\text { tes-1 }\end{array}$ & $\begin{array}{c}\text { Diaman- } \\
\text { tes-10 }\end{array}$ & $\begin{array}{c}\text { Diaman- } \\
\text { tes-20 }\end{array}$ \\
\hline & & & & \\
3 & $0,16 \mathrm{a}^{*}$ & $0,32 \mathrm{a}$ & $0,32 \mathrm{a}$ & $0,20 \mathrm{a}$ \\
6 & $1,00 \mathrm{~b}^{*}$ & $2,36 \mathrm{a}$ & $2,76 \mathrm{a}$ & $1,92 \mathrm{a}$ \\
9 & $3,80 \mathrm{~b}$ & $5,40 \mathrm{a}$ & $5,08 \mathrm{a}$ & $4,12 \mathrm{~b}$ \\
12 & $6,40 \mathrm{~b}$ & $7,80 \mathrm{a}$ & $7,36 \mathrm{a}$ & $5,84 \mathrm{c}$ \\
\hline
\end{tabular}

* $\mathrm{a}, \mathrm{b}=$ Valores medios con letra distinta en líneas horizontales son estadísticamente diferentes, Duncan $(\mathrm{P} \leq 0,05)$.

presentó además el menor desarrollo en las plantas (Cuadro 3) y fue la que mostró la menor abundancia de insectos (Cuadro 2). En general estos valores altos en el número promedio de $M$. hemipterus/planta, se podría deber a la cercanía (400 m) de una vieja plantación comercial infestada con estos escarabajos.

En el caso de $R$. palmarum su abundancia relativa fue menor, debido a que tiene un período de desarrollo larval más largo que el $M$. hemipterus y a que los restos de cosecha se descomponen rápidamente, antes de que se complete el desarrollo. El insecto completa con éxito el desarrollo en pejibaye para palmito cuando infesta la base de la planta, donde el tejido es más abundante y permanece vivo después de la cosecha. Mexzón (1995), encontró densidades de $M$. hemipterus de 1,6 y 2,4 insectos por palmera en las variedades sin espinas Diamantes-10 y Diamantes-1 respectivamente, en plantas para producción de fruta y manifiesta que este insecto podría convertirse en una plaga cuando se siembren comercialmente plantaciones de pejibaye sin espinas, por lo que sugiere, muestrear periódicamente e integrar varias medidas de combate como el uso de feromonas de atracción sexual, trampas con cebo alimentario con un insecticida, la eliminación de las vainas de las hojas secas y en casos especiales, quizás la aplicación de insecticidas a los residuos de cosecha dentro de la plantación.

\section{Defoliadores}

Un total de 60 insectos defoliadores fueron encontrados. Tres especies eran de saltamontes: $17(27,7 \%)$ Neoconocephalus sp. (Tettigoniidae); 16 (27,1\%) Conocephalus sp. (Acrididae) y $16(25,7 \%)$ Schistocerca pos. nitens (Acrididae); y 12 (19,5\%) del lepidóptero Saliana pos. severus Mabille (Hesperiidae) (Cuadro 1). La abundancia de estos defoliadores fue escasa en los primeros meses después de la siembra del cultivo y luego aumentó entre los seis y nueve meses, para decaer ligeramente a los 12 meses (Cuadro 1). Estos saltamontes cumplen parte de su desarrollo ninfal en el $P$. fasciculatum donde se camuflan con el follaje, para luego saltar al cultivo. Las especies fueron más abundantes en pejibaye sin espinas, debido a que plantas más vigorosas, proporcionan mejores condiciones de microclima. Asimismo, el combate de malezas obligó a los saltamontes a migrar al cultivo. 
Los saltamontes se alimentan de las hojas guías (flechas), haciendo surcos transversales y cuando la lámina foliar se expande, se observa una hilera transversal de agujeros y produce el quiebre de la lámina (Mexzón 1995). En un agroecosistema bien equilibrado, existen enemigos naturales que controlan sus poblaciones, como el hongo Hirsutella sp. (Figura 2), que coloniza al saltamontes y sus hifas crecen cubriendo completamente su cuerpo hasta aniquilarlo. Especies de Hirsutella, como por ejemplo H. citriformis Speare (Moniliales: Stilbaceae) han sido informados infectando cicadélidos y saltamontes (Shepard et al. 1987). También son comunes las avispas Evaniidae y Scelionidae; parasitan los huevos de estos saltamontes y así regulan sus poblaciones (Mexzón 1995).

Saliana sp. fue el defoliador más abundante a partir de los seis meses de edad de la planta, sin embargo, la herbivoría por larvas individuales no causó ningún daño económico. Esta mariposa tiene un amplio complejo de enemigos naturales, en su mayoría son avispas parasitoides, que parasitan los huevos, las larvas y las pupas (Mezxón y Chinchilla 1997).

Al igual que los saltamontes, Saliana sp. se presentó con mayor abundancia en las variedades sin espinas en comparación con la variedad con espinas (Cuadros 1 y 2, Figura 3) por causa de las condiciones de microclima anotadas. Si bien, en este estudio no se hicieron mediciones de los factores microclimáticos dentro de la plantación, existe una clara diferencia entre las variedades sin espinas al compararla con la variedad con espinas.

Saliana sp. es un defoliador común en la palma aceitera (Elaeis guineensis Jacquin) en América tropi$\mathrm{cal}$, con larvas solitarias que prefieren sitios protegidos

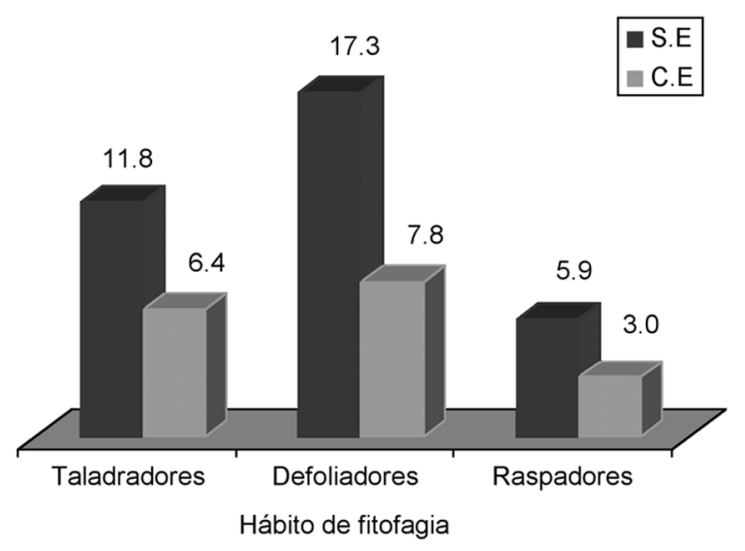

Figura 3. Distribución promedio de insectos $/ 10$ plantas y su hábito de fitofagia durante el primer año después de la siembra en pejibaye sin (S.E) y con (C.E) espinas. Guápiles, Costa Rica. 2001). de la radiación solar. En Costa Rica las mariposas adultas son comunes en Stachytarpheta cayenensis L. (Verbenaceae), planta que es común en los potreros cercanos al área de estudio y que explica la presencia de las larvas en el follaje del pejibaye (Mexzón 1997).

\section{Raspadores de follaje}

Un total de 21 insectos con el hábito de raspar el follaje del pejibaye fueron anotados. Demotispa pos. pallida estuvo representada por $16(76,2 \%)$ individuos y cinco insectos $(23,8 \%)$ eran de la especie Cephaloleia sp. Ambos se presentaron en menor abundancia que los taladradores y defoliadores (Figura 4), debido probablemente a lo limitado del sustrato alimenticio. Demotispa sp. se alimenta de la hoja guía cuando inicia su expansión y la abandona cuando ésta se ha completado.

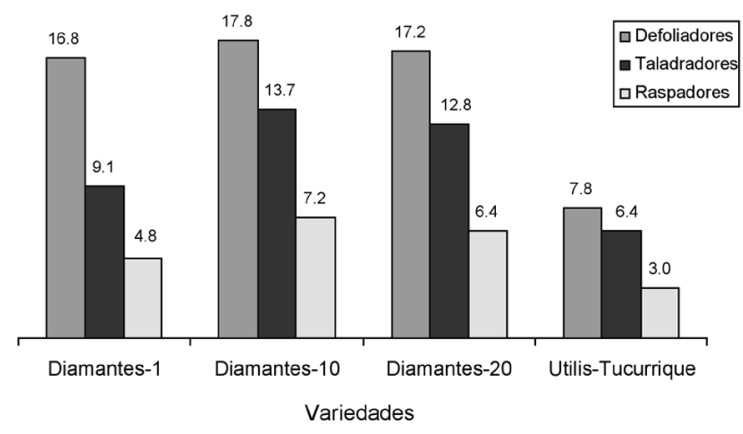

Figura 4. Número promedio de insectos/10 plantas, agrupados por el hábito de fitofagia en cuatro variedades de pejibaye durante el primer año de crecimiento. Guápiles, Costa Rica. 2001.

Mexzón (1995) informa que el número de Demotispa sp. encontrado oscila entre 15 a 25 insectos/hoja, sin embargo, en este estudio se encontraron bajas densidades de ambas especies, presentándose mayor abundancia en las variedades sin espinas que en aquella con espinas (Cuadros 1 y 2). En general su baja densidad se puede atribuir al parasitismo tan fuerte que ejerce una especie de hongo entomopatógeno (Metarhizium) durante los meses lluviosos. En el caso de Cephaloleia sp. tiende a ser escaso, porque solo se presenta en sitios poco perturbados. El insecto busca lugares donde las palmas traslapan su follaje y hay poca luminosidad (Mexzón 2001).

En este sentido, estos insectos prefieren las plantas de follaje denso y una mayor cantidad de hijuelos porque proporcionan mejores condiciones de microclima, como sucede en las variedades Diamantes-10 y Diamantes-20 en comparación con Diamantes-1 y Utilis-Tucurrique (Cuadro 2). Estas condiciones de microclima se podrían 
originar debido a la arquitectura de las variedades sin espinas, que son más vigorosas y muestran una mayor altura y número de hojas y rebrotes (Arroyo y Mora 2003) (Cuadros 3 y 4). Lo anterior es sugerido cuando se comparan las abundancias relativas de las especies de insectos en esas variedades (Figura 4).

En orden descendente y según el tipo de fitofagia, los defoliadores (Neoconocephalus sp., Conocephalus sp y Schistocerca pos nitens) fueron más abundantes que los taladradores del tallo (M. hemipterus y $R$. palmarum) y éstos a su vez fueron más abundantes que los raspadores del follaje (Cephaloleia sp. y Demotispa sp.) (Figura 4). Es importante destacar que este mismo orden se presentó en las cuatro variedades de pejibaye. Esto guarda relación con la etapa fenológica del cultivo, durante los primeros meses de desarrollo, los insectos fitófagos generalistas utilizan la planta de pejibaye como refugio y substrato de alimentación o reproducción, ya que un follaje denso y altura baja de las plantas, proporciona condiciones de microclima (temperatura más baja y mayor humedad relativa) más adecuadas. En este primer año de desarrollo conforme las plantas alcanzan una mayor altura, el número de especies tiende a incrementar (Mexzón 2004. Ob. per.).

\section{Otros}

Otro insecto que causa problema en las plantaciones de pejibaye y que aún no se ha estudiado detalladamente en el cultivo, es la hormiga Solenopsis geminata (Formicidae), que hace sus nidos en la base de la palmera y en las axilas o vainas de las hojas bajeras. La abundancia de hormigas fue menor a la edad de tres meses de plántulas en las variedades Diamantes-20 y Utilis-Tucurrique, mientras que la mayor abundancia relativa se presentó a los 12 meses y se obtuvo una diferencia significativa entre la variedad con espinas, Utilis-Tucurrique que presentó mayor abundancia que las variedades sin espinas (Cuadro 5).

Cuadro 5. Número promedio de hormigueros (Solenopsis geminata) en cuatro variedades de pejibaye, durante los 12 primeros meses de desarrollo. Guápiles, Costa Rica. 2001.

\begin{tabular}{lllll}
\hline $\begin{array}{c}\text { Edad del } \\
\text { palmito }\end{array}$ & \multicolumn{4}{c}{ Variedades } \\
\cline { 2 - 5 } & $\begin{array}{c}\text { Utilis- } \\
\text { Tucurrique }\end{array}$ & $\begin{array}{c}\text { Diaman- } \\
\text { tes-1 }\end{array}$ & $\begin{array}{c}\text { Diaman- } \\
\text { tes-10 }\end{array}$ & $\begin{array}{c}\text { Diaman- } \\
\text { tes-20 }\end{array}$ \\
\hline 3 meses & 0,0 & 0,0 & 0,2 & 0,2 \\
6 meses & $0,4 \mathrm{~b}$ & $0,4 \mathrm{ab}$ & $0,4 \mathrm{ab}$ & $0,8 \mathrm{a}$ \\
9 meses & $0,6 \mathrm{~b}$ & $0,6 \mathrm{~b}$ & $0,6 \mathrm{~b}$ & $1,0 \mathrm{a}$ \\
12 meses & $0,4 \mathrm{~b}$ & $0,8 \mathrm{~b}$ & $0,6 \mathrm{~b}$ & $1,2 \mathrm{a}$ \\
\hline
\end{tabular}

ab: Valores con diferente letra en sentido horizontal difieren estadísticamente, Duncan $(\mathrm{P} \leq 0,05)$.
S. geminata se asocia con el pseudocóccido Dysmicoccus brevipes L., al cual distribuye, para beneficiarse con las secreciones azucaradas (ligamaza) que éste excreta. La hormiga es más abundante en el pejibaye con espinas, porque las espinas proporcionan soporte físico para la construcción de su nido, además, la vaina no se desprende con facilidad, como sucede con las variedades sin espinas. También las espinas posiblemente proporcionan una barrera física contra depredadores y parasitoides (Mexzón 2003. Ob.per.). En este estudio $D$. brevipes no fue incluido en los muestreos periódicos.

En general de las nueve especies de insectos que se encontraron, ninguna alcanzó la condición de plaga y no afectó notoriamente el rendimiento productivo debido a que las poblaciones mostraron una densidad baja, quizás por el tamaño pequeño del área del cultivo y la presencia de los enemigos naturales. En este sentido Mexzón (1997, 1999 y 2001) manifiesta que muchos enemigos naturales de los insectos habitan en el follaje del pejibaye y en la vegetación natural que se localiza los alrededores de la plantación de palmito, lo que facilitó un equilibrio natural de los insectos fitófagos sin permitirles alcanzar el nivel de plaga para el cultivo.

Se señala la necesidad de un muestreo periódico de los insectos en las plantaciones de pejibaye para determinar las fluctuaciones poblacionales y tomar las medidas fitosanitarias anticipadas que sean necesarias. También se aconseja establecer medidas de manejo de ciertas especies de malezas en las cuales se alimenta la entomofauna, como una forma de atraer y mantener poblaciones de enemigos naturales de los insectos fitófagos.

\section{AGRADECIMIENTO}

Los autores agradecen al Ing. Edgar Vargas González, del laboratorio de Fitopatología de la Universidad de Costa Rica su colaboración en la identificación del hongo Hirsutella sp.

\section{LITERATURA CITADA}

ARROYO, C.; MORA, J. 2002. Producción comparativa de palmito entre cuatro variedades de pejibaye (Bactris gasipaes Kunth). Agronomía Mesoamericana 13(2): 135-140.

ARROYO, C.; MORA, J. 2003. Aspectos fenológicos del desarrollo en pejibaye (Bactris gasipaes Kunth) para palmito. Agronomía Mesoamericana. 14(2): 165-176. 
BOGANTES, A. 2003. Evaluación de 4 distancias de siembra y 2 estrategias de control de malezas en pejibaye ( $\mathrm{Bac}$ tris gasipaes Kunth) sin espinas para palmito. Tesis Magíster Scientiae. Facultad de Ciencias Agroalimentarias. Universidad de Costa Rica. San José. 106 p.

CHINCHILLA, C. M. 2003. Manejo integrado de problemas fitosanitarios en palma aceitera (Elaeis guineensis) en América Central. Manejo Integrado de Plagas y Agroecología 67: 69-82.

COUTURIER, G.; CLEMENT, C. R.; FILHO, V. 1991. Leptoglossus lonchoides Allen (Heteroptera, Coreidae), causante de la caída de los frutos de Bactris gasipaes (Palmae) en la Amazonia Central. Turrialba 41(3): 293-298.

MEXZÓN, G. R.; CHINCHILLA, C. M.; CASTRILLO, G.; SALAMANCA, D. 1994. Biología y hábitos de Rhynchophorus palmarum L. asociado a la palma aceitera en Costa Rica. ASD Oil Palm Papers 8:14-21.

MEXZÓN, G. R. 1995. Control natural de los artrópodos perjudiciales en pejibaye. In: García, J.; Fuentes, G.; Monge, J. (eds.). Opciones al uso unilateral de plaguicidas en Costa Rica. San José, Costa Rica. Editorial EUNED. p. 157-163.

MEXZÓN, G. R. 1997. Pautas de manejo de las malezas para incrementar las poblaciones de insectos benéficos en el cultivo de palma aceitera (Elaeis guineensis Jac.). Agronomía Mesoamericana 8(2): 21-32.

MEXZÓN, G. R.; CHINCHILLA, C. M. 1997. Enemigos naturales de los artrópodos perjudiciales en palma aceitera en América tropical. ASD Oil Palm Papers 13: 9-33.

MEXZÓN, G. R. 1999. Manejo integrado de los artrópodos perjudiciales. In: J. Mora-Urpí.; J. Gainza (eds.). Palmito de pejibaye (Bactris gasipaes Kunth) su cultivo e industrialización. San José, Costa Rica, Editorial Universidad de Costa Rica. p.138-147.
MEXZÓN, G. R. 2001. Manejo ecológico de plagas. Curso práctico sobre manejo ecológico de plagas y enfermedades. Ambio-Fundación/Universidad EARTH, Limón, Costa Rica. p.1-45.

MORA, J.; VARGAS, E.; LÓPEZ, C. A.: VILLAPLANA, M.; ALLÓN, G.; BLANCO, C. 1982. El pejibaye. Publicación del Banco Nacional de Costa Rica. Universidad de Costa Rica. San José, Costa Rica. 16 p.

O'BRIEN, C. W.; KOVARIK, P. W. 2000. A new genus and new species of weevil infesting fruits of the palm ( $\mathrm{Bac}$ tris gasipaes H.B.K) (Coleoptera: Curculionidae). Coleopterists Bulletin 54(4): 459-465.

PEÑA, R. E. 2000. Plagas y enfermedades del chontaduro. In: R. Cuesta.; E. Peña.; J. Gómez (eds.). Manual técnico $\mathrm{N}^{\circ}$ 4. El cultivo de chontaduro (Bactris gasipaes K) para palmito. Tumaco, Colombia. Publicación de CORPOICA REGIONAL CINCO. p.73 - 84.

ROTHSCHUH, J. 1983. Guía técnica para el cultivo del pijay (Bactris gasipaes H.B.K.). IICA. Fondo Simón Bolívar. IICA: Serie de publicaciones misceláneas. $\mathrm{N}^{\mathrm{o}}$ 445. Managua, Nicaragua. 24p.

SAS. Statystical Análisis System. 2001. SAS Institute Inc. Cary, N.C, USA. Release 8.2.

VASQUEZ, J.; O'BRIEN, CH. W.; COUTURIER, G. 2000. Dynamis nitidulus (Coleoptera:Curculionidae), nueva plaga del pejibaye. Manejo Integrado de Plagas. $\mathrm{N}^{\circ} 58$ : 70-72.

VELAZCO, A. 1991. Chontaduro (Bactris gasipaes). Investigaciones realizadas en Colombia durante el período 1980 - 1990. In: J. Mora; J. Gainza (eds.). Palmito de pejibaye (Bactris gasipaes Kunth) su cultivo e industrialización. San José, Costa Rica, Editorial Universidad de Costa Rica. pp. 287- 300. 